\title{
Sachregister Vol. 37,1997
}

Adnextumoren 5 Amnioninfektionssyndrom 143 Androstenon 150 Antike geburtshilfliche Lehre 154 Azidoserate 191

Biometrie 21

CerbB-2-Antikörper 54

Diabetische Schwangere 21 Docetaxel 54 Doppler-Sonographie 203 Ductus venosus 203

Ektope Schwangerschaft 209 Externe Qualitätssicherung 14 Extrauteringravidität 62

Fetalblut 130

Fetale Herzinsuffizienz 203

Oxygenierung 130

Physiologie 130 Fetaler Hyperinsulinismus 21

Säure-Basen-Status 130 Fetales Hörempfinden 119

Schicksal 203 Fibrinkleber 62 Forzepsrate 191 Fruchtwasserinsulin 21

Gastroschisis 25 Gebärstuhl 154 Geburt 154 Gemcitabin 54

Hysteroplastik 48 Hochdosischemotherapie 54

Intrauterine retroamniale Abszedierung

143

Kaiserschnitt 25 Kolposkopie 179 Kopuline 150 Kordozentese 130

Laparoskopie 5, 62

Lärmschädigung in der Schwangerschaft

119 Ligatur der Arteria uterina 41 Lymphom 68

Mamma 68 Mammakarzinom 54 -, brusterhaltende Therapie 136 Mayer-Rokitansky-Küster-Syndrom 44 Menstruation 30 Menstruationshygiene 30

Mikroblutgasanalyse 191 Mittelalterliche geburtshilñiche Lehre 154 Mortalität 191

Negatives Gram-Präparat aus Fruchtwasser

143 Neue endokrine Medikamente 54 Neues Gesamttherapiekonzept, Ovarial-

karzinom 221 Non-Hodgkin-Lymphom 68

Onkoplastische Rekonstruktion, ältere

Frau 136 Operative Gynäkologie 14 Ovarialkarzinom(e) 216 -, Figo-Stadien III-IV 221

Paclitaxel 54

Papillomvirusinfektion 179 Pheromone 150

Platinhaltige Polychemotherapie 221 Präoperative Diagnostik 5 Prognostische Faktoren, Ovarialkarzinom 221

Quadrantektomie 136

Reproduktion 150

Schwangerschaft nach Arterienligatur 41 Schwangerschaftsrate 62 Sectiorate 191

Second-look-Laparoskopie 39 Sterilität 48 Systemische Therapie 54

Topotecan 54 Tuba uterina 216 Tubenkarzinom 216 Tubenperforation 39 Tuboskop 39

Uteruserhaltende Massnahmen 209 Uterine Missbildungen 48

Vaginalaplasie 44 Vaginale Chirurgie 44 - Geburt 25 Vaginalsonographie 209 Vakuumextraktionsrate 191 Versteckte Ovulation 150 Vibroakustische

Stimulationstests 119 Vinorelbin 54

Weckreiz 119 Werbung 30

Zervikale intraepitheliale Neoplasie 179 Zervikalschwangerschaft 209 Zytologie 179 Zytotoxisches Arzneimittelexanthem 143

KAIUiER

E-Mail karger@kargcr.ch Fax+ 41613061234 http://www.karger.ch

(C) 1998S. KargerAG, Basel

This article is also accessible online at: http://BioMedNet.com/karger 